\title{
Massive Alveolar Haemorrhage Presenting During the COVID-19 Pandemic
}

Authors:

Disclosure:

Acknowledgements:

Received:

Accepted:

Keywords:

Citation:
*Joseph Page, Caitlin Morgan, John Hughes, Janet Fallon

Respiratory Medicine Department, Musgrove Park Hospital, Taunton and Somerset Foundation Trust, Taunton, UK

*Correspondence to joseph.page1@nhs.net

The authors have declared no conflicts of interest.

All authors treated the patient subject to the case report. Dr Page and Dr Morgan drafted the manuscript and Dr Fallon provided critical appraisal. Dr Hughes kindly provided the images. All authors reviewed and approved the final version of the manuscript.

13.11.20

08.01.21

Anti-neutrophil cytoplasmic antibody (ANCA)-positive vasculitis, coronavirus disease (COVID-19), haemoptysis, pulmonary haemorrhage.

EMJ Respir. 2021; DOI/10.33590/emjrespir/20-00267.

\section{Abstract}

The coronavirus disease (COVID-19) pandemic has challenged healthcare systems and has resulted in complex diagnostic processes for patients with non-COVID-19 pathology. Here, we demonstrate a case of massive alveolar haemorrhage secondary to antineutrophil cytoplasmic antibody-positive vasculitis, presented to a district general hospital in the UK during the first wave of the global pandemic. This case highlights some of the difficulties clinicians may face when diagnosing lifethreatening antineutrophil cytoplasmic antibody-positive vasculitis amidst the COVID-19 pandemic. The authors place emphasis on the careful interpretation of chemical biomarkers such as troponin and D-dimer when assessing patients with acute respiratory distress. They also aim to highlight the importance of CT thorax imaging when seeking an alternate diagnosis to COVID-19.

\section{BACKGROUND}

The coronavirus disease (COVID-19) pandemic has challenged healthcare systems and has resulted in complex diagnostic processes for patients with non-COVID-19 pathologies. Recent data have shown that elevated troponins in patients with COVID-19 are related to cardiac injury and poor prognosis., ${ }^{1,2}$ However, it is not clear whether these biological markers are secondary to the respiratory distress associated with COVID-19 or to the primary acute cardiac disease. ${ }^{3}$
The authors demonstrate a case of massive alveolar haemorrhage secondary to antineutrophil cytoplasmic antibody (ANCA)positive vasculitis, which presented to a district general hospital during the COVID-19 pandemic. The patient was treated successfully with plasma exchange and immunosuppressive therapy after exclusion of COVID-19. The case highlights the difficulty in diagnosing and treating patients with non-COVID-19 pathology during the pandemic, with emphasis on radiological imaging. 


\section{CASE PRESENTATION}

A 61-year-old male presented with acute dyspnoea, cough, and haemoptysis. The patient had a history of Type 2 diabetes mellitus with retinopathy, hypertension, previous deep vein thrombosis, and hypercholesterolaemia. He was no longer taking anticoagulants. He also reported haematuria 2 weeks prior to admission.

An initial chest radiograph raised concern of possible COVID-19, showing centrally diffuse bilateral ground-glass opacifications. Admission biochemistry revealed: haemoglobin 70 $\mathrm{g} / \mathrm{L}$; eosinophils $0.36 \times 10^{9} / \mathrm{L}$; troponin 2,385 $\mathrm{ng} / \mathrm{L} ; \quad \mathrm{D}$-dimer $0.65 \mathrm{ug} / \mathrm{mL}$; and erythrocyte sedimentation rate of $45 \mathrm{~mm} /$ hour. An ECG was suggestive of left ventricular hypertrophy and dynamic ST segment changes in V1-V3. He was transfused two units of packed red cells and proceeded to a CT scan of the thorax. This confirmed central opacification and bilateral ground-glass shadowing in the lungs without evidence of pulmonary embolus (Figure 1A). The report suggested the possibility of COVID-19 or vasculitis as a differential; therefore, the patient remained in respiratory isolation until two COVID-19 nasopharyngeal swabs were negative and further biochemical testing was performed.

Vasculitis screening soon confirmed ANCA positivity $(>1 / 320)$ with positive myeloperoxidase levels (>8.0 apolipoprotein). The patient was urgently reviewed by the rheumatology team and started on induction immunosuppressive therapy: cyclophosphamide with pulsed methylprednisolone. Thereafter, the patient continued a maintenance dose of prednisolone at $60 \mathrm{mg}$ daily. One week following cyclophosphamide, the patient experienced increasing episodes of chest pain associated with dyspnoea, tachycardia, and fever. He was treated with empirical antibiotics for suspected hospitalacquired pneumonia and transferred back to the respiratory isolation ward, while COVID-19 infection was further excluded.

He was transfusion dependent to maintain a haemoglobin level of $>70 \mathrm{~g} / \mathrm{L}$ and continued to experience large-volume haemoptysis with persisting hypoxia. His renal function deteriorated with a 30\% rise in creatinine $(163 \mathrm{mmol} / \mathrm{L})$. Repeat ECG showed further ischaemic changes in V1-V3. His case was discussed with the cardiology department who advised on further cardiac imaging and management of a Type 2 myocardial infarction secondary to ongoing pulmonary haemorrhage.

Cardiac MRI revealed severe left ventricular impairment without late gadolinium enhancement. Repeat CT thorax demonstrated new areas of ground-glass density and consolidation, consistent with progressive alveolar haemorrhage (Figure 1B).

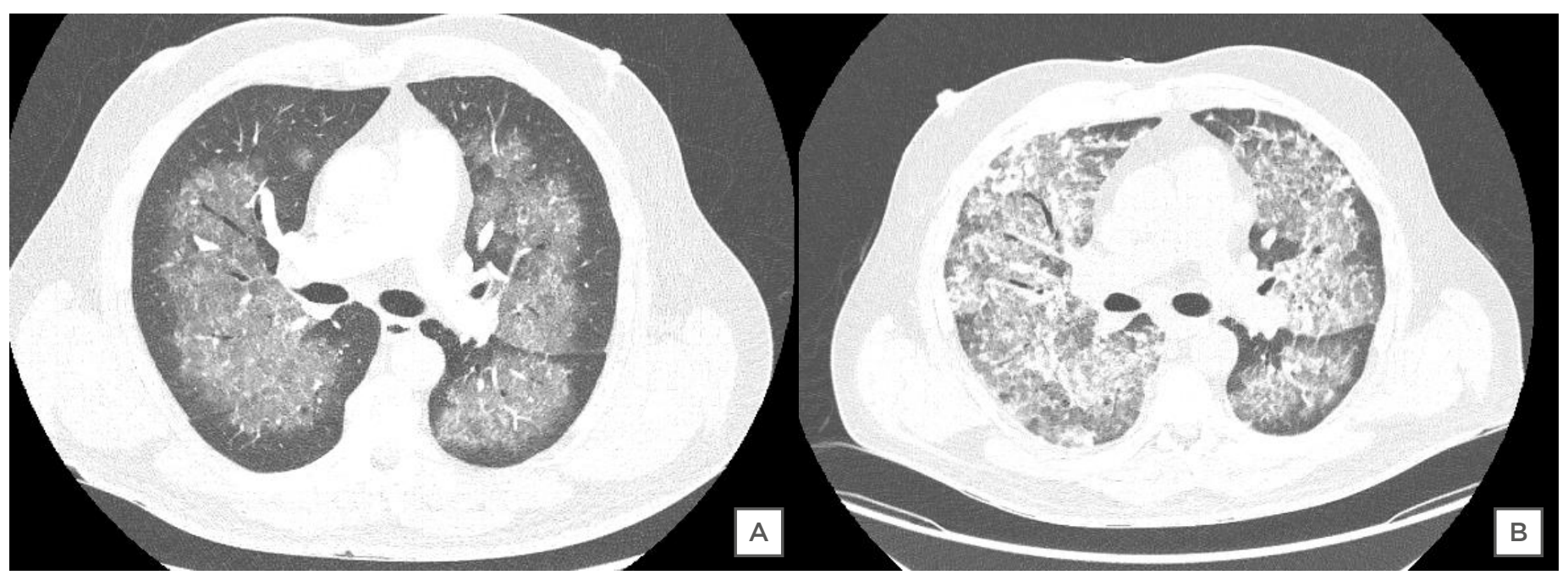

Figure 1: A) CT thorax image confirming central opacification and ground-glass shadowing in the lungs bilaterally; B) comparative CT thorax image at Day 24 confirming new areas of ground-glass density and consolidation consistent with progressive alveolar haemorrhage. 
Following further discussion with the rheumatologists, the patient was transferred to the high dependency unit for seven cycles of plasma exchange. The patient clinically improved throughout his plasma exchange without further need for blood transfusion or oxygen therapy. He denied any further episodes of chest pain and was successfully discharged after 39 days in the hospital.

\section{Outcome and Follow-up}

The patient subsequently received invasive angiography for non-ST-elevation myocardial infarction. The left anterior descending artery had severe, mid-vessel disease, which was successfully stented during his induction therapy. A followup chest radiograph confirmed resolution of the initial pulmonary haemorrhage. After discussion with the cardiology, rheumatology, and respiratory medicine departments, the patient remains on dual antiplatelet therapy. He awaits a decision regarding coronary artery bypass grafting following completion of his sixth cycle of cyclophosphamide infusions.

\section{DISCUSSION}

The authors have demonstrated a case of massive pulmonary haemorrhage secondary to ANCApositive vasculitis that presented to a district general hospital during the COVID-19 pandemic. The clinical picture was typical of vasculitis given the patient's history of haematuria, haemoptysis, and associated hypoxaemia. However, initial diagnosis was challenging for two reasons: firstly, there were diagnostic algorithms implemented in response to the global pandemic to ensure appropriate levels of patient safety; secondly, the prodromal overlap and patient characteristics between ANCA-positive vasculitis and COVID-19 further complicated the diagnosis. ${ }^{4,5}$

ANCA-positive vasculitides are the most common small-vessel vasculitides and include microscopic polyangiitis, eosinophilic granulomatosis with polyangiitis (Churg-Strauss Syndrome), and granulomatosis with polyangiitis (Wegener's granulomatosis). They are frequently missed or delayed in their diagnosis due to their nonspecific symptoms. Clinicians may further attribute specific disease symptoms to more prevalent conditions such as viral pneumonia and asthma. ${ }^{6-8}$ The most commonly reported prodromal symptoms of ANCA-positive vasculitides and COVID-19 were dyspnoea, fever, and cough. ${ }^{4,9}$ In one study, the median delay from initial symptom onset to a diagnosis of ANCApositive vasculitis (or relapse) was significantly longer during the pandemic than previous cohorts. ${ }^{4}$ A key distinguishing feature between ANCA-positive vasculitis and COVID-19 infection is the presence of haemoptysis. The literature suggests that haemoptysis is uncommon in COVID-19 and has a symptom prevalence of $2 \% .^{10,11}$ Therefore, the presence of haemoptysis is likely to indicate an alternate respiratory pathology rather than COVID-19 infection.

Although ANCA-positive vasculitis remains a rare cause for haemoptysis in the UK, both granulomatosis with polyangiitis and microscopic polyangiitis can present with large-volume haemoptysis because of the associated alveolar haemorrhage.12,13 It is essential that clinicians consider these vasculitides in their differential diagnosis due to an untreated mortality of almost $90 \%$ at 2 years. ${ }^{14}$ The triad of haemoptysis, acute hypoxic respiratory failure, and diffuse lung infiltrates should further point towards the possibility of diffuse alveolar haemorrhage. This is a life-threatening manifestation of ANCA-positive vasculitis and requires prompt induction immunosuppressive therapy. However, there is substantial debate as to whether treatment with plasma exchange leads to better patient outcomes. ${ }^{15,16}$

Early bronchoalveolar lavage (BAL) is the diagnostic test needed to confirm diffuse alveolar haemorrhage..$^{17}$ It is also needed to exclude both infection (i.e., pneumocystis) and malignancy from the differential. The sequential rise in red blood cells or haemosiderin-laden macrophages on repeat BAL is considered a diagnostic marker. However, BAL was not performed in this case for several reasons. Firstly, the patient's clinical deterioration meant that it was not safe to perform bronchoscopy. Secondly, the procedure is highly aerosol-generating, and current guidance suggests it should only be performed in the most necessary of cases to minimise the potential risk of COVID-19 transmission. ${ }^{18}$ Thirdly, BAL has limited value in determining the specific aetiology of diffuse alveolar haemorrhage. The clinical presentation, positive biochemistry results, and multidisciplinary team approach 
within this case provided consensus that the radiological imaging represented diffuse alveolar haemorrhage rather than COVID-19 or any other pathology.

Individuals with myeloperoxidase ANCApositive vasculitis have a mean age of 59 years at diagnosis ( \pm 17.6 years standard deviation) and a slight male predominance. ${ }^{19}$ From the hospital's COVID-19 database (April 2020), the mean age of patients testing positive for the virus was 68 years and almost two-thirds were male $(n=63 / 105) .{ }^{20}$ Moreover, COVID-19 and ANCApositive vasculitis patients have similar preexisting comorbidities; hypertension, diabetes, cardiovascular disease, and obesity are frequently reported comorbidities associated with COVID-19-related death. ${ }^{1,2,9}$ Similarly, there is a significantly increased risk of cardiovascular mortality in patients with myeloperoxidase ANCA-positive vasculitis. ${ }^{21}$ This is in part due to disease activity as well as some of the side effects caused by immunosuppressive therapy. It is proposed that risk factors for cardiovascular disease are monitored closely and that medications for hypertension, diabetes, and hypercholesterolaemia are optimised in ANCApositive patients..$^{21,22}$

The pandemic has placed great importance on triaging patients with symptoms of acute respiratory distress syndrome and isolating them in dedicated wards while awaiting nasopharyngeal test results. The use of reversetranscriptase (RT)-PCR for nucleic acid detection of COVID-19 is currently considered to be the gold-standard diagnostic test. However, RTPCR testing is not without its shortcomings; one study suggested that up to $54 \%$ of patients may produce an initial false-negative test for COVID-19 infection with RT-PCR. ${ }^{23}$ If there is strong clinical suspicion for COVID-19, the patient should undergo repeat nasopharyngeal RTPCR testing and remain in respiratory isolation due to the risks associated with false-negative results. ${ }^{24}$ Therefore, the patient received two nasopharyngeal RT-PCR tests and remained in respiratory isolation until further biochemical and radiological information was available.

The pandemic has also placed emphasis on the early evaluation of poor prognostic markers in suspected patients with COVID-19 using cardiac biomarkers and D-dimer. ${ }^{1-3}$ High D-dimer results are shown to correlate with disease severity and mortality in COVID-19.25 However, these biomarkers are nonspecific and have an unknown role in the management of COVID-19. The use of D-dimer also raises considerable questions relating to coagulopathy and venousthromboembolic disease in COVID-19. Expert guidance advises against the use of D-dimer in the diagnostic evaluation for venousthromboembolic disease of suspected patients with COVID-19. ${ }^{26}$ It is pertinent to remember that these positive biomarkers may relate to other serious disease processes such as acute myocardial infarction, pulmonary embolism, and diffuse alveolar haemorrhage.

The chest radiograph findings in diffuse alveolar haemorrhage and COVID-19 are nonspecific and require further characterisation with CT thorax. The classic cross-sectional imaging findings in COVID-19 and diffuse alveolar haemorrhage include ground-glass opacities (GGO) with or without consolidation. ${ }^{9,27}$ However, it is the pattern of disease that helps clinicians differentiate between the two diagnoses. The GGO and consolidation seen in ANCAassociated diffuse alveolar haemorrhage are usually widespread and bilateral with a perihilar predominance. Typically, the mid and lower zones of the lung are affected, with subpleural and apical sparing. ${ }^{28,29}$ Dense consolidation typically represents complete filling of the alveoli with acute blood.30 This leads to interlobular septal thickening with GGO creating a 'crazypaving' pattern. These changes may resolve within 10-14 days or progress to interstitial fibrosis with repeated haemorrhage. 12,13,27,30 The repeat $\mathrm{CT}$ imaging (Figure $1 \mathrm{~B}$ ) in this case clearly demonstrates consolidation of varying density, which suggests repeated and progressive alveolar haemorrhage.

When assessing CT imaging of the chest in COVID-19 it is not just the pattern of GGO and consolidation that differs but its temporal relationship with symptom onset. CT thorax in the presymptomatic and early disease phase (0-2 days) may be normal. Therefore, CT imaging is not a reliable standalone investigation to exclude early COVID-19.31 Most patients with COVID-19 will typically develop bilateral, multilobar lung involvement with a diffuse, peripheral and subpleural distribution. These radiological changes are predominately GGO during the first 
week of COVID-19 presentation and progress to mixed GGO with dense consolidation in a reverse halo sign over the next 2 weeks. ${ }^{32}$ Patients with COVID-19 further develop a fibrotic picture with reticulation and irregular interlobular septal thickening. However, it is currently unknown whether this is fully reversible. The distinction between the pattern of the two diseases combined with the patient's negative RT-PCR test was fundamental in seeking an alternate diagnosis to COVID-19.

\section{CONCLUSION}

This case demonstrates the added complexity of diagnosing rarer causes of diffuse alveolar haemorrhage during the global pandemic. Haemoptysis is an uncommon symptom in
COVID-19 and should prompt clinicians to consider other diagnoses for acute respiratory distress. CT thorax imaging was fundamental in seeking an alternate diagnosis to COVID-19. The authors hope this case report highlights the difference in patterns of GGO and consolidation between COVID-19 and pulmonary manifestations of ANCA-positive vasculitis. Although the use of plasma exchange in ANCApositive vasculitis is debatable, the authors feel that the repeat imaging supported a diagnosis of possible induction treatment failure and expedited the need for plasma exchange. This case also highlights the pertinence of cautious interpretation of biochemistry results, particularly with reference to troponin and D-dimer in the context of acute respiratory distress.

Patient consent for publication: Obtained.

\section{References}

1. Zhou F et al. Clinical course and risk factors for mortality of adult inpatients with COVID-19 in Wuhan, China: a retrospective cohort study. Lancet. 2020;395(10229):1054-62.

2. Guo T et al. Cardiovascular implications of fatal outcomes of patients with coronavirus disease 2019 (COVID-19). JAMA Cardiol. 2020;5(7):811-8.

3. Shi S et al. Association of cardiac injury with mortality in hospitalized patients with COVID-19 in Wuhan, China. JAMA Cardiol. 2020;5(7):80210

4. Giollo A et al. Challenge of diagnosing ANCA-associated vasculitis during COVID-19 pandemic: a missed 'window of opportunity'. Ann Rheum Dis. 2020;DOI:10.1136/ annrheumdis-2020-218830.

5. Shenavandeh S et al. COVID-19 and granulomatosis with polyangiitis (GPA): a diagnostic challenge. Rheumatology. 2020;59(8):2170-1.

6. Hunter R et al. ANCA associated vasculitis. BMJ. 2020;369:m1070.

7. Suresh E. Diagnostic approach to patients with suspected vasculitis Postgrad Med J. 2006;82:483-8.

8. Yates M, Watts R. ANCA associated vasculitis. Clin Med (London). 2017;17(1):60-4.

9. Konopka $\mathrm{K}$ et al. Diffuse alveolar damage (DAD) resulting from coronavirus disease 2019 infections is morphologically indistinguishable from other causes of DAD. Histopathology. 2020;77(4):570-8.
10. Fu L et al. Clinical characteristics of coronavirus disease 2019 (COVID-19) in China: a systematic review and meta-analysis. J Infect. 2020;80(6): 656-65.

11. Peys E et al. Haemoptysis as the first presentation of COVID-19: a case report. BMC Pulmon Med. 2020;20:275

12. Castaner $\mathrm{E}$ et al. When to suspect pulmonary vasculitis: radiological and clinical clues. Radiographics. 2010;30(1):33-53.

13. Marten $\mathrm{K}$ et al. Pattern-based differential diagnosis in pulmonary vasculitis using volumetric CT. AJR Am J Roentgenol. 2005;184:720-33

14. Fauci AS et al. Wegener's granulomatosis: prospective clinical and therapeutic experience with 85 patients for 21 years. Ann Intern Med. 1983:98:76-85

15. Klemmer PJ et al. Plasmapheresis therapy for diffuse alveolar haemorrhage in patients with small vessel vasculitis. Am J Kidney Dis. 2003;42(6):1149.

16. Walsh $M$ et al. Plasma exchange and glucocorticoids in severe ANCAassociated vasculitis. N Eng J Med. 2020;382:622-31.

17. Park M. Diffuse alveolar haemorrhage Tuberc Respir Dis. 2013;74(4):151-62.

18. Wahidi $M$ et al. The use of bronchoscopy during the coronavirus disease 2019 pandemic: CHEST/ AABIP guideline and expert panel report. Chest. 2020;158(3):1268-81.

19. Charles Jennette J, Nachman P. ANCA glomerulonephritis and vasculitis. CJASN. 2017;12(10):1680-91.

20. Arnold B et al. Musgrove Park Hospital COVID-19 dataset April 2020. Presented at Medical Grand round. July 2020.

21. Wallace ZS et al. Cardiovascular disease is the most common cause of death in ANCA associated vasculitis (AAV). Rheumatology (Oxford). 2019;58(Suppl 52)S65-9.

22. Kronbichler A et al. Comorbidities in ANCA-associated vasculitis. Rheumatology. 2020;59:79-83.

23. Arevalo-Rodriguez I et al. Falsenegative results of initial RTPCR assays for COVID-19: a systematic review. medRxiv. 2020;15(12):e0242958.

24. Watson $\mathrm{J}$ et al. Interpreting a COVID-19 test result. BMJ. 2020;369:1-7.

25. Yao $Y$ et al. D-dimer as a biomarker for disease severity and mortality in COVID-19 patients: a case control study. J Intensive Care. 2020;8(49):111.

26. Moore $L$ et al. Prevention, diagnosis, and treatment of VTE in patients with coronavirus disease 2019: guideline and expert panel report. Chest. 2020;158(3):1143-63

27. Castaner $E$ et al. Imaging findings in pulmonary vasculitis. Semin Ultrasound CT MR. 2012;33(6):567-79.

28. Sanchez-Oro R et al. Radiological findings for diagnosis of SARS-CoV-2 pneumonia (COVID-19). Med Clin (Barc). 2020;155:36-40.

29. Chung $M$ et al. Imaging of pulmonary 
vasculitis. 2010;255(2):322-34.

30. Primack S, et al. Diffuse pulmonary haemorrhage: clinical, pathological, and imaging features. AJR Am J Roentgenol. 1995;164:295-300.
31. Bernheim $A$ et al. Chest CT findings in coronavirus disease 2019 (COVID-19): relationship to duration of infection. Radiology. 2020;295(3):685-91.
32. Shi $\mathrm{H}$ et al. Radiological findings from 81 patients with COVID-19 pneumonia in Wuhan, China: a descriptive study. Lancet Infect Dis. 2020;20:425-34. 